\title{
Curva de estimativa das internações hospitalares nas regiões metropolitanas brasileiras: um estudo de série histórica'
}

Estimation curve of hospital admissions in Brazilian metropolitan regions: a historical series study

Curva estimativa de internaciones hospitalarias en las regiones metropolitanas brasileñas: un estudio de serie histórica

Graziella Denúbila Gomes ${ }^{1}$, Carolina Marques Borges², Raquel Costa Rossete Melo³, Ana Cristina Viana Campos ${ }^{4}$

\section{RESUMO}

O objetivo deste estudo foi investigar as variações temporais na distribuição das internações hospitalares realizadas no Sistema Único de Saúde (SUS) em todas as Regiões Metropolitanas brasileiras entre os anos de 1995 e 2006. Trata-se de estudo ecológico de série histórica com dados secundários referentes às 36 regiões metropolitanas brasileiras. Realizou-se a análise descritiva dos dados, correlação de Spearman e curva de estimativa. Os resultados indicam que o Teste $F$ foi significativo em ordem decrescente para os modelos linear $(p=0,001)$, quadrático $(p=0,002)$ e cúbico $(p=0,004)$. Por outro lado, os valores de $R^{2}$ apresentaram ordem crescente de valores - 0,277; 0,307 e 0,338 , respectivamente. O modelo cúbico mostrou que o percentual médio de internações hospitalares em 2006 apresentou tendência de queda quando comparado ao percentual médio de internações no ano de 1995. Conclui-se que o percentual de internações hospitalares realizadas no SUS nas regiões metropolitanas brasileiras decresceu durante o período analisado.

Descritores: Hospitalização; Admissão do Paciente; Serviços de Informação; Sistema Único de Saúde; Epidemiologia.

\section{ABSTRACT}

The objective of this study was to investigate temporal variations in the distribution of hospital admissions occurring in the National Health System (SUS) in all Brazilian metropolitan areas between 1995 and 2006. This ecological, historical series study was performed using secondary data from 36 Brazilian metropolitan regions. We conducted a descriptive analysis of the data, and then subjected the data to Spearman correlation and curve estimation. The results indicate that the $F$ test was significant in a decreasing order for the linear ( $p=0.001)$, quadratic $(p=0.002)$ and cubic $(p=0.004)$ models. On the other hand, the $R^{2}$ values showed an increasing order of values $(0.277,0.307$ and 0.338$)$, respectively. The cubic model showed that the mean percentage of hospital admissions in 2006 declined when compared to 1995. In conclusion, the percentage of admissions occurring in the SUS in Brazilian metropolitan areas decreased during the studied period.

Descriptors: Hospitalization; Patient Admission; Information Services; Single Health System; Epidemiology.

\section{RESUMEN}

Se objetivó investigar las variaciones temporales en la distribución de internaciones hospitalarias efectuadas en el Sistema Único de Salud (SUS) en todas las Regiones Metropolitanas brasileñas entre 1995 y 2006. Estudio ecológico de serie histórica con datos secundarios referentes a las 36 regiones metropolitanas brasileñas. Se realizó análisis descriptivo de datos, correlación de Spearman y curva de estimación. Los resultados indican que el Test F fue significativo en orden decreciente para los modelos lineal ( $p=0,001)$, cuadrático $(p=0,002)$ y cúbico $(p=0,004)$. Por otro lado, los valores de $R^{2}$ presentan orden creciente de valores $(0,277 ; 0,307$ y 0,338 , respectivamente). El modelo cúbico mostró que el porcentaje promedio de internaciones hospitalarias en 2006 presentó tendencia de caída comparado con el porcentual promedio de internaciones de 1995. Se concluye en que el porcentaje de internaciones hospitalarias realizadas en el SUS en regiones metropolitanas brasileñas decreció durante el período analizado.

Descriptores: Hospitalización; Admisión del Paciente; Servicios de Información; Sistema Único de Salud; Epidemiologia.

\footnotetext{
I Trabalho apresentado ao Instituto de Educação Continuada da Pontifícia Universidade Católica de Minas Gerais (IEC-PUC Minas) para obtenção do título de especialista em Enfermagem Terapia Intensiva.

${ }_{1}^{1}$ Enfermeira, Especialista em Enfermagem Terapia Intensiva. Belo Horizonte, MG, Brasil. E-mail: gdenubila@yahoo.com.br.

2 Odontologa, Mestre em Saúde Pública, Discente do Programa de Pós-Graduação em Odontologia - nível Doutorado, Faculdade de Odontologia (FO), Universidade Federal de Minas Gerais (UFMG). Belo Horizonte, MG, Brasil. E-mail: carolmborges@yahoo.com.br.

3 Odontologa, Discente do Programa de Pós-Graduação em Odontologia - nível Mestrado, FO, UFMG. Belo Horizonte, MG, Brasil. E-mail: raquelrossette@yahoo.com.br.

${ }^{4}$ Odontologa, Mestre em Odontologia, Discente do Programa de Pós-Graduação em Odontologia - nível Doutorado, FO, UFMG. Belo Horizonte, MG, Brasil. E-mail: campos.acv@gmail.com.
} 


\section{INTRODUÇÃO}

O Sistema de Informação Hospitalar (SIH) é o sistema de mapeamento de internação hospitalar no âmbito público, sendo que as internações custeadas diretamente ou cobertas por seguro-saúde não são contadas. O laudo médico é o documento utilizado para solicitar a internação do paciente em hospitais que prestam atendimento ao SIH/SUS, devendo ser corretamente preenchido em todos os seus campos. Permite a emissão de AlH que irá garantir o acesso ao estabelecimento hospitalar, bem como o pagamento dos serviços de saúde prestados aos usuários do SUS, durante a internação hospitalar ${ }^{(1)}$.

O SIH reúne informações sobre $70 \%$ das internações hospitalares do país e é alimentado mensalmente pelos formulários de Autorização de Internação Hospitalar (AlH) que contém informações sobre o paciente e sobre os procedimentos realizados, bem como a autorização do pagamento pela internação hospitalar ${ }^{(2-3)}$.

Alguns estudos apresentam significativas diferenças entre as taxas de internação hospitalar devido às diferentes afecções, bem como relacionadas ao tempo de internação ${ }^{(4-6)}$. Além disso, este é um indicador que pode ser fortemente influenciado também por fatores socioeconômicos, epidemiológicos e demográficos, infraestrutura de serviços, bem como por políticas públicas assistenciais e preventivas ${ }^{(7)}$.

A apropriação dessas informações pelos gestores e pela sociedade pode servir de subsídio para o planejamento e desenvolvimento de ações que busquem reduzir as desigualdades no adoecer e no acesso aos serviços de saúde ${ }^{(8)}$. Por outro lado, a utilização de serviços hospitalares no Brasil é motivada pela necessidade de saúde, sendo este um fenômeno incomensurável. Os indicadores de necessidade de saúde geralmente são baseados em morbidade referida e, dessa forma, diferem do resultado de uma avaliação clínica ${ }^{(9)}$.

Para isso se faz necessário a realização de avaliações regulares e sistemáticas dos dados disponibilizados, a fim de demonstrar a fragilidade dos dados e maior necessidade de aprimoramento no registro dessas informações(10).

O presente artigo teve como objetivo investigar as variações temporais na distribuição das internações hospitalares realizadas no Sistema Único de Saúde nas
Regiões Metropolitanas brasileiras entre os anos de 1995 a 2006.

\section{MÉTODOS}

Trata-se de um estudo ecológico de série histórica com utilização de dados secundários obtidos no mês de março do ano de 2009 na página eletrônica do banco de dados do $\mathrm{SIH}$, gerados e consolidados pelo Departamento de Informática do Sistema Único de Saúde - DATASUS em cooperação com o Centro Nacional de Epidemiologia (CENEPI) ${ }^{(11)}$.

A amostra foi constituída pelas 36 regiões metropolitanas (RM) brasileiras com dados disponíveis: RM de Belo Horizonte, Colar de Belo Horizonte, RM do Vale do Aço, Colar do Vale do Aço, RM de Vitória, RM do Rio de Janeiro, RM de São Paulo, RM da Baixada Santista, RM de Campinas, RM de Curitiba, RM de Londrina, RM de Maringá, RM de Florianópolis, Expansão de Florianópolis, RM do Vale do Itajaí, Expansão do Vale do Itajaí, RM do Norte/Nordeste Catarinense, Expansão do Norte/Nordeste Catarinense, RM da Foz do Itajaí, Expansão da Foz do Itajaí, RM Carbonífera, Expansão da Carbonífera, RM de Tubarão, Expansão de Tubarão, RM de Porto Alegre, RM de Goiânia e Entorno de Brasília.

$\mathrm{O}$ indicador considerado para o presente estudo foi o percentual médio de internações hospitalares, calculado pelo número total de internações hospitalares de residentes pagas pelo SUS multiplicado por 100, dividido pela população total residente de cada ano(7).

Inicialmente realizou-se a estatística descritiva do número de internações para os anos de 1995 a 2006. Em seguida, considerou-se como variável dependente o número de internações hospitalares em 2006. Esta foi comparada ao número de internações de todos os outros anos (1995 a 2005) pela Correlação de Spearman.

Finalmente, utilizou-se a Curva de Estimativa para estimar parâmetros de regressão e produzir modelos de relação linear, quadrática e cúbica entre o número médio de internação em 2006 (variável denominada de "y") e o número médio de internação em 1995 (variável denominada " $x$ "). Essas duas variáveis foram escolhidas por representarem o maior intervalo de tempo entre os dados.

O banco de dados foi construído no programa estatístico Statistical Package for Social Sciences for Windows - SPSS versão com nível de significância estabelecido em $p<0,05$. 


\section{RESULTADOS}

A média do percentual de internações hospitalares para as regiões metropolitanas apresenta tendência de queda, passando de 7,88 em 1995 para 5,95 em 2006, com ligeiro aumento entre os anos de 1998 e 1999. Em 11 anos pode-se observar também que a diferença entre os valores máximos e mínimos reduziu pela metade (Tabela 1).

Tabela 1: Medidas de resumo para o percentual médio de internações hospitalares nas Regiões Metropolitanas brasileiras entre os anos de 1995 e 2006 . DATASUS, 2009.

\begin{tabular}{|c|c|c|c|c|c|c|}
\hline Ano & Mínimo & Mediana & Média & Desvio padrão & Máximo & Correlação \\
\hline 1995 & 3,46 & 7,77 & 7,88 & 2,10 & 12,36 & 0,571 \\
\hline 1996 & 4,41 & 7,35 & 7,49 & 1,59 & 10,60 & 0,642 \\
\hline 1997 & 4,16 & 7,15 & 7,36 & 1,69 & 13,02 & 0,673 \\
\hline 1998 & 4,44 & 7,27 & 7,38 & 1,64 & 13,97 & 0,747 \\
\hline 1999 & 4,58 & 7,64 & 7,55 & 1,44 & 12,24 & 0,777 \\
\hline 2000 & 4,52 & 7,40 & 7,21 & 1,36 & 12,01 & 0,841 \\
\hline 2001 & 4,27 & 7,15 & 6,94 & 1,38 & 11,92 & 0,887 \\
\hline 2002 & 4,38 & 6,93 & 6,74 & 1,30 & 10,76 & 0,895 \\
\hline 2003 & 4,56 & 6,63 & 6,49 & 1,06 & 9,06 & 0,905 \\
\hline 2004 & 4,55 & 6,45 & 6,27 & 0,88 & 8,48 & 0,906 \\
\hline 2005 & 4,23 & 6,24 & 6,15 & 0,80 & 8,14 & 0,897 \\
\hline 2006 & 3,90 & 6,12 & 5,95 & 0,84 & 7,91 & $1,00^{\mathrm{b}}$ \\
\hline
\end{tabular}

${ }^{a} p<0,001$

b valor de referência para correlação de Spearman

O resultado da correlação foi estaticamente significante entre todas as variáveis $(p<0,001)$ e indicam que a relação entre o número de consultas é mais forte quanto mais próximo do ano de 2006, com exceção do ano de $2005(0,897)$ (Tabela 1$)$.
Os resultados do Teste $\mathrm{F}$ foram significativos em ordem decrescente para os modelos linear $(p=0,001)$, quadrático $(p=0,002)$ e cúbico $(p=0,004)$. Por outro lado, os valores de $\mathrm{R}^{2}$ apresentaram ordem crescente de valores $(0,277 ; 0,307$ e 0,338) (Tabela 2$)$.

Tabela 2. Resumo da análise dos modelos Linear, Quadrático e Cúbico para o número de internações hospitalares em 1995 e 2006. DATASUS, 2009.

\begin{tabular}{cccccccc}
\hline \multirow{2}{*}{ Equação } & \multicolumn{3}{c}{ Modelo } & \multicolumn{5}{c}{ Estimativas dos parâmetros } \\
\cline { 2 - 6 } & $\mathrm{R}^{2}$ & $\mathrm{~F}$ & $\mathrm{p}$-valor & Constante & $\mathrm{b}^{1}$ & $\mathrm{~b}^{2}$ & - \\
\hline Linear & 0,277 & 13,051 & 0,001 & 4,286 & 0,211 & - & - \\
Quadrático & 0,307 & 7,317 & 0,002 & 2,552 & 0,671 & $-0,029$ & - \\
Cúbico & 0,338 & 5,446 & 0,004 & 6,990 & $-1,204$ & 0,220 & $-0,010$ \\
\hline
\end{tabular}

O modelo cúbico mostra que o percentual médio de internações hospitalares em 2006 apresenta tendência de queda em relação ao percentual médio de internações no ano de 1995. A construção desse modelo pode ser representada pela seguinte equação: $y=6,990$ $+(-1,204) x+0,220 x^{2}+(-0,010) x^{3}$ (Tabela 2).

O gráfico de curva (Gráfico 1) apresenta o ajuste de cada modelo com os valores observados para todas as regiões metropolitanas, sendo que o modelo cúbico parece ser o mais adequado, pois segue melhor o formato da distribuição dos dados. 
Gráfico 1: Percentual de internações hospitalares das Regiões Metropolitanas brasileiras de acordo com os ajustes dos modelos Linear, Quadrático e Cúbico.

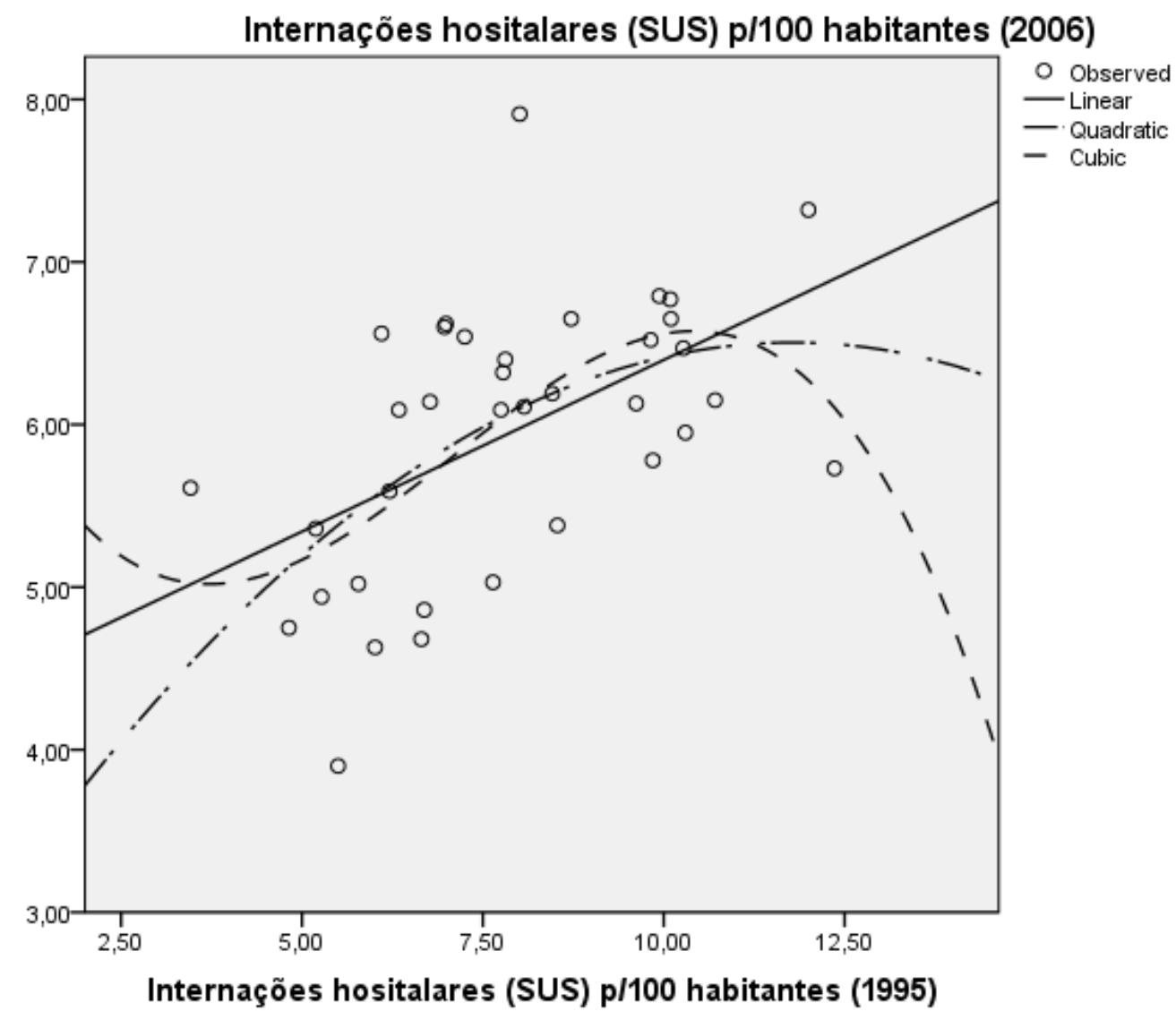

\section{DISCUSSÃO}

A correlação entre o número de internações hospitalares aparentemente pareceu ser positiva, ou seja, quanto maior o ano de referência maior os coeficientes de correlação. Entretanto, a média de internações por habitante diminuiu entre os anos de 1995 a 2006, existindo uma relação negativa, e uma curva descreveria melhor essa relação do que uma reta.

Para o país como um todo, observa-se uma tendência irregular de queda no número de internações hospitalares nas grandes regiões. No Norte e CentroOeste, há um aumento do número de internações por habitante até 1999, declinando a partir de então e, a região Sul apresentava o maior número de internações por habitante. A partir de 2003 , esta posição é assumida pela região Centro-Oeste. O menor número de internações por habitante ocorre na região Sudeste, em todos os anos, com exceção de 1995, quando a região Norte apresentava esta característica(7). Entretanto, somente $3 \%$ da variação da utilização das admissões hospitalares podem ser atribuídos às diferenças na oferta de serviços de saúde entre os Estados brasileiros ${ }^{(12)}$.

Na verdade, se percebe que existe uma associação com maior número de internações e desigualdades sociais, desigualdades no uso dos serviços públicos de saúde ${ }^{(9)}$. Possivelmente a correlação encontrada no presente estudo também seja mais sensível a estes fatores do que simplesmente ao fator tempo.

Faz-se importante ressaltar que os estudos de tendência que avaliam o comportamento temporal do percentual de internação hospitalar, não nos permitem incluir outros fatores importantes a essas relações tais como: mudanças na estrutura demográfica, baixa resolutividade dos serviços básicos, inserção dos hospitais no sistema de saúde, incorporação tecnológica, papel do gestor no sistema local de saúde ${ }^{(13)}$.

Neste estudo, a associação entre as variáveis foi fraca, sendo mínima a diferença entre os modelos cúbico e linear. A estatística de correlação $\left(R^{2}\right)$ é uma medida da força da associação entre os valores observados e modelos de previsão da variável dependente, sendo que grandes valores de $\mathrm{R}^{2}$ indicam relações fortes. 
O modelo linear apresentou maior significância no Teste $F(p=0,001)$, entretanto os resultados dos outros testes apontam que este é o pior modelo para explicar a variabilidade no número de internações hospitalares. Por outro lado, apesar de o modelo cúbico ter apresentado maior eficiência de estimativa, há uma forma clara para os casos (Regiões Metropolitanas) do gráfico que parece não ser acompanhado pela parcela de ajuste da curva dos modelos, o que significa que existe um padrão nos dados que não é captado por nenhum dos modelos.

$O$ valor de significância da estatística $F$ foi inferior a 0,05 para todos os modelos. Isso significa que a variação explicada pelos modelos não é devido ao acaso, portanto os resultados são válidos para o período considerado. Esses resultados podem indicar o quanto se faz necessário melhor acompanhamento e planejamento, a fim de reduzir os gastos de materiais de consumo, equipamentos, medicamentos e recursos ${ }^{(14)}$.

Os estudos com dados extraídos do DATASUS vêm sendo amplamente utilizados, entretanto o fato desses dados estarem separados por períodos determinados, sem nenhuma orientação sobre como agregar diferentes períodos, torna-se difícil a confiabilidade dos estudos de tendências ${ }^{(15-16)}$.

Além disso, os problemas relacionados à utilização de dados secundários são conhecidos na literatura. Um dos maiores problema de confiabilidade dos dados do SIH/SUS é a seletividade do registro das internações por não incorporar as internações hospitalares custeadas por recursos privados. Isso significa que é possível estabelecer tendências e padrões das morbidades somente para a população assistida pelo SUS ${ }^{(13)}$. Podemse destacar problemas relacionados ao registro do diagnóstico na internação, em não apresentar informações completas, e às fraudes para aumentar o reembolso financeiro das internações ${ }^{(12,17)}$.

Para a vigilância epidemiológica da doença pneumocócica, a análise dos dados do SIH tem potencial para avaliar o impacto do programa de vacinação ${ }^{(18)}$. Para outras doenças, como a doença hemolítica perinatal ainda não parece ser recomendável(19).

Apesar das limitações, a utilização destas ferramentas gera informações que servem para caracterizar o panorama estadual/nacional das condições de saúde e monitorar as condições de morbidades e mortalidades hospitalares. Além disso, poderá identificar problemas na captação e registro de dados, fornecendo subsídios para o planejamento, programação e avaliação de políticas públicas de saúde ${ }^{(20)}$.

Outra limitação deste estudo foi ter utilizado dados de regiões metropolitanas que inclui as capitais, onde há maior concentração do número de estabelecimentos de saúde, de disponibilidade de recursos humanos e terapêuticos. Por outro lado, quanto maior a dimensão geográfica que o dado secundário abrange, maior a compilação das informações, maior a chance de comparabilidade entre as regiões em questão. Ainda assim, justamente por serem grandes centros urbanos é importante avaliar com maior cuidado a efetividade das políticas públicas do SUS que primam por melhores níveis de saúde e qualidade de vida da população.

\section{CONCLUSÃO}

Pode-se concluir que houve uma tendência de diminuição do percentual médio de internações hospitalares no período considerado para a maioria das regiões metropolitanas investigadas.

Por outro lado, algumas regiões não se encaixaram em nenhum modelo explicativo, podendo indicar que existem outros fatores importantes que explicam esse comportamento, tais como diferenças socioeconômicas dos municípios em questão. Possivelmente, com uma amostra maior de municípios e informações mais consistentes, seja possível produzir evidências mais detalhadas acerca do comportamento dessa tendência de diminuição do número de internações hospitalares.

Um dos maiores problemas para confiabilidade das informações de saúde é a sub-informação. Faz-se necessário conscientizar os profissionais responsáveis pelo correto preenchimento das fichas clínicas/planilhas e pela vigilância sobre os responsáveis que transmitem esses dados para o sistema. Assim, a internação hospitalar pode ser um bom indicador no entendimento das diferenças existentes entre as regiões metropolitanas brasileiras, necessitando-se de mais estudos com os dados disponíveis no DATASUS. 


\section{REFERÊNCIAS}

1. Ministério da Saúde. Secretaria de Atenção à Saúde. Departamento de Regulação, Avaliação e Controle. Manual técnico do Sistema de Informação Hospitalar / Ministério da Saúde, Secretaria de Atenção à Saúde, Departamento de Regulação, Avaliação e Controle. - Brasília : Editora do Ministério da Saúde. [Internet] 2007. [cited 2011 oct 25]. (Série A. Normas e Manuais Técnicos). Available from:

http://bvsms.saude.gov.br/bvs/publicacoes/07_0066_M.pdf 2. Cavalini LT, Leon ACMP. Correção de sub-registros de óbitos e proporção de internações por causas mal definidas. Rev Saúde Pública. 2007;41(1):85-93.

3. Mathias TAF, Soboll MLMS. Confiabilidade de diagnósticos nos formulários de autorização de internação hospitalar. Rev. Saúde Pública. 1998;32(6):526-32.

4. Caminal HJ, Starfi B, Sánchez RE, Hermosilla PE, Martín MM. La atención primaria de salud y las hospitalizaciones por ambulatory care sensitive conditions en Cataluña. Rev Clin Esp. 2001;201(9):5017.

5. Niti M, Ng TP. Avoidable hospitalisation rates in Singapore, 19911998: assessing trends and inequities of quality in primary care. J Epidemiol Community Health. 2003;57(1):17-22.

6. Motta CCR, Hansel CG, Silva J. Perfil de internações de pessoas idosas em um hospital público. Rev. Eletr. Enf. [Internet]. 2010 [cited 2010 oct 01];12(3):471-7. Available from:

http://www.fen.ufg.br/revista/v12/n3/v12n3a08.htm.

7. Ministério da Saúde. [Internet]. Brasília: Organização PanAmericana da Saúde. Rede Interagencial de Informação para a Saúde. Indicadores básicos para a saúde no Brasil: conceitos e aplicações 2008. [cited 2010 oct 01]. Available from:

http://tabnet.datasus.gov.br/cgi/idb2006/indicadores.pdf. 8. Garbinato LR, Béria JU, Figueiredo ACL, Raymann B, Gigante LP, Palazzo LS, et al. Prevalência de internação hospitalar e fatores associados: um estudo de base populacional em um centro urbano no Sul do Brasil. Cad Saúde Pública. 2007;23(1):217-24.

9. Castro MSM, Travassos C, Carvalho MS. Fatores associados às internações hospitalares no Brasil. Ciênc. saúde coletiva. 2002;7(4):795-811.

10. Lima CRA, Scharamm JMA, Coeli CM, Silva MEM. Revisão das dimensões de qualidade dos dados e métodos aplicados na avaliação dos sistemas de informação em saúde. Cad Saúde Pública. 2009;25(10):2095-09.

11. DATASUS [Internet]. Brasília: Ministério da Saúde (BR) Departamento de Informática do SUS - DATASUS. [cited 2010 oct 01]. Available from: http://w3.datasus.gov.br/datasus/datasus.php. 12. Castro MSM, Travassos C, Carvalho MS. Efeito da oferta de serviços de saúde no uso de internações hospitalares no Brasil. Rev Saúde Pública. 2005;39(2):277-84.

13. Chaves LDP, Anselmi ML. Explanatory factors for the variation in hospitalizations. Rev Latino-Am Enfermagem. 2008;16(2):287-92. 14. Lemos C, Chaves LDP, Azevedo ALCS. Sistemas de informação hospitalar no âmbito do SUS: revisão integrativa de pesquisas. Rev. Eletr. Enf. [Internet]. 2010 [cited 2010 oct 01];12(1):177-85.

Available from:

http://www.fen.ufg.br/revista/v12/n1/v12n1a22.htm.

15. Candiago RH, Belmonte-de-Abreu P. Uso do Datasus para avaliação dos padrões das internações psiquiátricas, Rio Grande do Sul. Rev Saúde Pública. 2007;41(5):821-29.

16. Góis ALB, Veras, RP. Informações sobre a morbidade hospitalar em idosos nas internações do Sistema Único de Saúde do Brasil. Ciênc. saúde coletiva. 2010;15(6):2859-69.

17. Oliveira TCR, Latorre MRDO. Tendências da internação e da mortalidade infantil por diarréia: Brasil, 1995 a 2005. Rev Saúde Pública. 2010;44(1):102-11.

18. Bittencourt SA, Camacho LAB, Leal MC. Hospital Information Systems and their application in public health. Cad Saude Publica. 2006;22(1):19-30.

19. Novaes HMD, Sartori AMC, Soárez PC. Hospitalization rates for pneumococcal disease in Brazil, 2004-2006. Rev Saúde Pública 2011;45(3):539-47.

20. Picolo GD, Chaves LDP, Azevedo ALCS. A produção científica sobre avaliação em serviços de internação hospitalar no Brasil: revisão integrativa. Rev. Eletr. Enf. [Internet]. 2009 [cited 2010 oct
01];11(2):395-402. Available from:

http://www.fen.ufg.br/revista/v11/n2/v11n2a22.htm.

Artigo recebido em 06.10.2010.

Aprovado para publicação em 13.03.2012.

Artigo publicado em 30.03.2012. 